\title{
Core-Collape Supernova Progenitors: Light-Curve and Stellar-Evolution Models
}

\author{
Melina C. Bersten \\ Instituto de Astrofísica de La Plata (IALP), CCT-CONICET-UNLP. Paseo del Bosque S/N \\ (B1900FWA), La Plata, and Facultad de Ciencias Astronómicas y Geofísicas, Universidad \\ Nacional de La Plata, Argentina, Kavli Institute for the Physics and Mathematics of the \\ Universe (WPI), The University of Tokyo, 5-1-5 Kashiwanoha, Kashiwa, Chiba 277-8583, \\ Japan \\ email: mbersten@f caglp.unlp.edu.ar
}

\begin{abstract}
A very active area of research in the field of core-collapse supernovae ( $\mathrm{SNe}$ ) is the study of their progenitors and the links with different subtypes. Direct identification using preand post-SN images is a powerful method but it can only be applied to the most nearby events. An alternative method is the hydrodynamical modeling of SN light curves and expansion velocities, which can serve to characterize the progenitor (e.g. mass and radius) and the explosion itself (e.g. explosion energy and radioactive yields). This latter methodology is particularly powerful when combined with stellar evolution calculations. We review our current understanding of the properties of normal core-collapse SNe based chiefly on these two methods.
\end{abstract}

Keywords. binaries: general, supernovae: general

\section{Introduction}

Most of massive stars $\left(M_{\mathrm{ZAMS}} \gtrsim 8 M_{\odot}\right)$ end their lives as core-collapse supernovae (CCSNe), leaving behind either neutron stars or black holes, and ejecting heavy elements into space with large explosion energies. Thus, these events are important in our understanding of the chemical and dynamical evolution of the Universe. Nonetheless, it is not yet clear what is the mechanism that transforms the core collapse into a SN explosion.

It has long been known that there are different types of CCSNe. The classification is mainly based on spectral properties. The main division comes from the presence (Type II) or absence (Type I) of hydrogen (H) lines in the spectra (Filippenko(1997)). Type II $\mathrm{SNe}$ (or H-rich) are the most common type of the explosion in the Universe, more than $50 \%$ of CCSNe belong to this Type (Smith et al. (2011)). In section 3.1 we provide an overview of our knowledge of these objects. Type I (or H-deficient) SNe are usually also called "Stripped-envelope SNe" (SESNe). Depending on the degree of envelope stripping, they are classified as Type IIb, Ib, or Ic. We review these objects in section 3.2 .

An important remaining problem in astrophysics is finding the links between SN Types and their progenitor stars. In particular, it is important to know what kind of CCSNe come from single stars, as opposed to interacting binary systems. Recent studies of open clusters have indicated that the incidence of interacting binaries among massive stars is particularly large (about 70\%; Sana et al. (2012)). This clearly shows the relevance of close binary evolution in connection with SN progenitors. There are also others arguments that support the idea that the majority of SESNe originate from binary systems. For example, the fractions of different SN Types (Smith et al. (2011)) and the ejecta mass estimates in SESNe (Drout et al. (2011), Lyman et al. (2014)). In particular for the case of SN IIb, 
there is further evidence of binarity with the detection of the companion star in late-time observations, as in the case of SN 1993J (Maund et al. (2004), Fox et al. (2014)) and possibly in SN 2011dh (Folatelli et al. (2014)).

\section{Progenitor Identification Methods}

There are several methods proposed in the literature to analyze physical properties of the SN progenitors, namely:

- Archival pre-explosion image searches (combined with post-explosion observations)

- Environmental and metallicity studies

- SN rates

- Mass-loss rates from radio and X-rays

- Spectropolarimetry

- Very early ("flash") spectroscopy

- Light-curve and spectrum modeling

The search for progenitor stars in deep pre-explosion images is a powerful, direct approach to understand the origin of SNe. It provides a critical test for stellar evolution models. Important results have been achieved using this technique, which we describe in section 2.1. However, in most cases, either because the SN is too distant or simply due to lack of pre-supernova images, other methods are required to infer progenitor properties. One such method is the hydrodynamical modeling of SN observations which we describe in section 2.2 .

\subsection{Archival imaging method}

This technique is based on the identification of a possible progenitor star using archival pre-explosion images of the SN location. The association then needs to be confirmed using post-explosion imaging to confirm the disappearance of the progenitor candidate. This method has been largely favored with the use of the HST archive. Important results have been found in recent years. Currently, there are 20 progenitor detections and $\approx 30$ upper limits (see Smartt (2015) for a review). In general terms, there are two main groups leading efforts in this area, one led by S. Smartt and the other by S. Van Dyk. Using pre-explosion photometry an estimate of the main-sequence mass of the progenitor can be derived by assuming some stellar evolution model. Below we list some of the main conclusions achieved with this method:

- Confirmation that Type II-P SNe arise from the explosion of red supergiant (RSG) stars, as previously predicted by stellar evolution calculations and by hydrodynamical analysis of SN light curves.

- SN II-P progenitors have $\mathrm{M}_{\mathrm{ZAMS}} \lesssim 16-18 \mathrm{M}_{\odot}$

- One blue supergigant (BSG) progenitor detected in association with the famous SN 1987A, a peculiar Type II SN.

- One Luminous Blue Variable (LBV) progenitor associated with a Type IIn SN. †

- Some yellow supergiant (YSG) progenitors detected, mostly associated with Type IIb SNe.

- No detection of Type Ib or Ic SN progenitors with the possible exception of SN iPTF13bvn (see more details in section 3.2)

$\dagger$ Type IIn SNe are objects that show narrow lines in their spectra. The narrow lines are indicative of interaction between the SN ejecta and a previously existing circunmstellar medium $(\mathrm{CSM})$. 


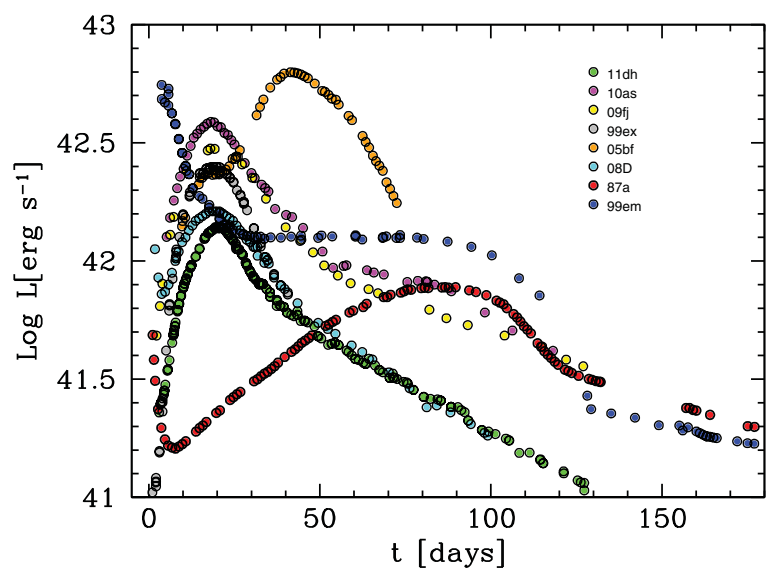

Figure 1. Bolometric light curves of CCSNe showing the diversity among these Types of SNe.

We emphasize that with the current technology, this method cannot be applied to events further than $\sim 30 \mathrm{Mpc}$. Therefore, more indirect methods are required to analyze the progenitors of the majority of SNe.

\subsection{Hydrodynamical modeling}

CCSNe light curves (LC) are very heterogeneous (see Figure 1), as opposed to the standard-candle Type Ia SN. This heterogeneity is associated to varying progenitor properties. It is a very well-known fact that the LC morphology is sensitive to the physical characteristics of the SN progenitor. Therefore, LC modeling, ideally combined with modeling of spectra or photospheric velocities, provides a useful way to constrain progenitor properties, such as mass and radius, as well as explosion parameters (explosion energy and production of radioactive material). This methodology is particularly powerful when combined with stellar evolution calculations.

It must be noted that there is currently no self-consistent model for the origin of the SN explosion. Nevertheless, SN problem is usually decoupled into two independent processes: the explosion trigger, and the ejection of the envelope. The propagation of the explosion through the envelope can be simulated independently of how it is triggered. In this way, it is possible to study the observational outcome of the explosion, such as LCs and spectra. Bersten et al. (2011) developed a one-dimensional Lagrangian code with flux-limited radiation diffusion and gray transfer for gamma-rays to artificially explode the hydrostatic structures and analyze the outcome after the shock propagation. Figure 2 shows how sensitive the LC is on the initial progenitor structure assumed. A typical SN II$\mathrm{P}$ LC is produced when one explodes a RSG structure. For BSG progenitors a typical $87 \mathrm{a}$-like $\mathrm{SN}$ is produced. If one adopts a compact progenitor, such as a helium (He) star, the typical ${ }^{56} \mathrm{Ni}$-powered LC is produced.

Different phases of the LC evolution can be distinguished, each one with different dependence on physical parameters (see Figure 3). For the case for RSG, with thick H envelopes, we can distinguish a cooling phase (mainly dependent on the stellar radius), a plateau phase (dependent on $\mathrm{H}$ mass, explosion energy and radius), and a tail phase (mainly determined by the radioactive material). For more compact structures, most of the SN evolution happens during the radioactive-power phase. However, the early emission provides unique information about the structure of the star previous to the explosion, as well as the mixing processes. The small progenitor radius is responsible for the rapid degradation of the shock energy, leading to a fast initial peak that is usually 

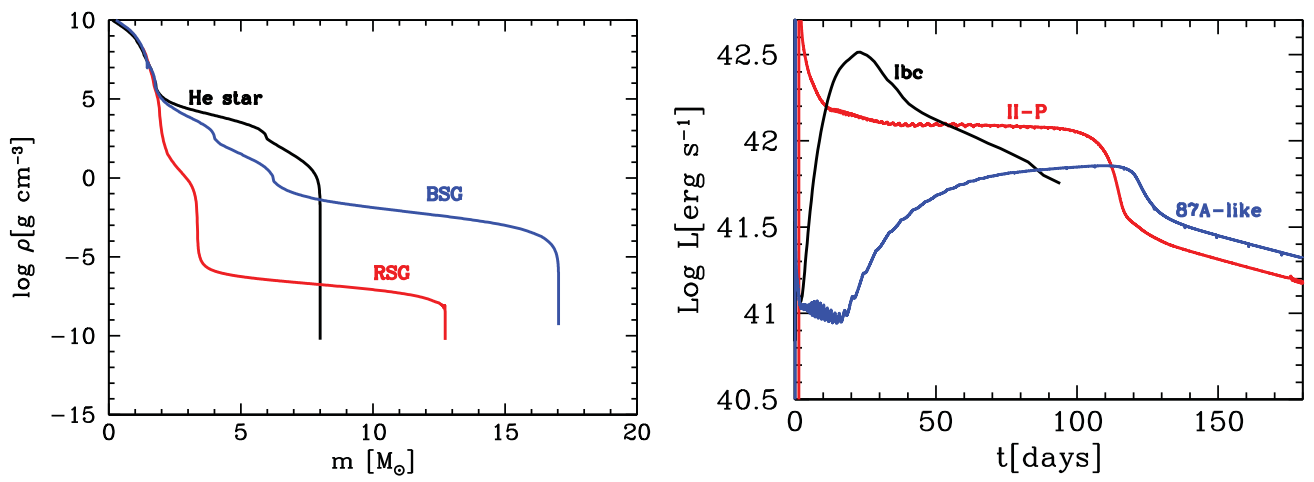

Figure 2. (Left) Progenitor structures before the explosion. (Right) Bolometric LCs for different progenitor structures assumed.
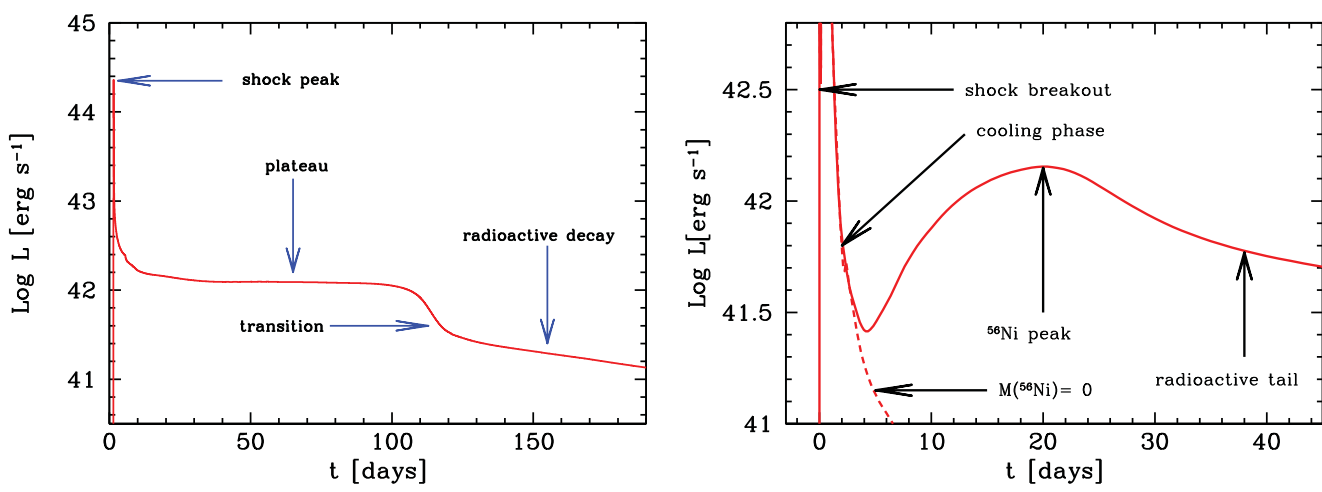

Figure 3. (Left) Bolometric LC for a RSG structure. (Right) Bolometric LC for SESNe. Different phases of LC evolution are indicated in the figures.

unobserved. Bersten et al. (2012) have shown the dependence of the early emission on the progenitor radius for Type IIb SNe (see their Figure 10), and for Type Ib SNe in Bersten et al. (2014) (see their Figure 2). Until recently, only a handful of SESNe were observed during the cooling phase. However, given the important information provided during this phase, large efforts are being made by current surveys to catch the $\mathrm{SNe}$ as early as possible. Therefore, it is expected to have an interesting progress in this area in coming years.

\section{SN Progenitors}

In the following section we briefly summarize some recent results on CCSNe progenitors, separated into H-rich and H-poor (or SESNe) events.

\subsection{Hydrogen-rich}

H-rich SNe are the most common type of explosion in nature, amounting to $\sim 50 \%$ of all CCSNe. Additional interest on SNe II-P has arisen from the fact that they have been proposed as good distance indicators, independent of Type Ia SNe, for example via the standard-candle method (SCM) (Hamuy \& Pinto (2002)). Regarding the range of masses of their progenitors, pre-explosion imaging connected with stellar evolution calculations suggest a range of masses of $M_{\mathrm{ZAMS}} 8-16 M_{\odot}$. This is in contradiction with hydrodynamical LC modeling (see for example Utrobin (2007)), which predicts a larger 


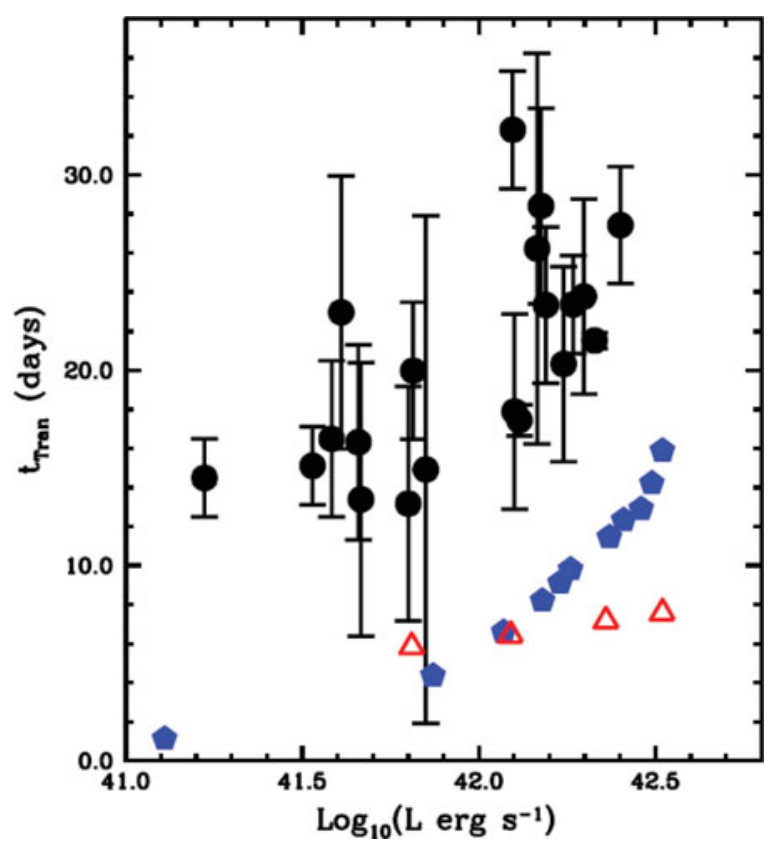

Figure 4. Bolometric luminosity at the mid-point of the plateau as a function of $t_{\text {trans }}$ for CSP data (black points) and for models with different radii (blue points) and different energies (red triangles)

range of masses, as noted by Smartt (2009). Until now, the reason of this discrepancy is not clear.

There are some recent works focused on the properties of the early LC. For example González-Gaitán et al. (2015) have shown that the typical radii of SNe II progenitors should be $\approx 500 \mathrm{R}_{\odot}$, or alternatively, some CSM needs to be present nearby. This conclusion was based on rise-time studies of a sample of early LC. Recent hydrodynamical modeling has also suggested the presence of CSM in order to explain the LC of some SNe II (Morozova et al. (2016)). Figure shows the relation between $t_{\text {trans }}$ (defined as the epoch of the beginning of the plateau phase) and the plateau luminosity (black points) for a large sample of very well-observed SNe II observed by the Carnegie Supernova Project (Anderson et al. (2014)). A set of hydro models is also shown as a comparison (blue points for different progenitor radii and red triangles for different explosion energies). Although the models can reproduce the dependency of the data there is a clear offset between models and observations. This can be solve by assuming some CSM close to the progenitor star (Anderson et al., prep). Our results indicate that most of the H-rich progenitors need to loose some material previous to the explosion, in concordance with previous works based on fewer objects.

Finally, it is important to mention that SNe II have recently been proposed as good metallicity indicators (Anderson et al. (2016)).

\subsection{Hydrogen-poor}

The two most appealing mechanisms to remove the $\mathrm{H}$ envelope necessary to produce SESNe progenitors are strong stellar winds in very massive stars $\left(M \gtrsim 25 M_{\odot}\right)$ and mass transfer in close binary systems (see Langer (2012) for a recent review). Pre-explosion image analysis as well as LC modeling suggest low mass progenitors (see e.g. Drout et al. (2011), Lyman et al. (2014)), which can only be reconciled with the binary scenario. 
In addition, there are three confirmed YSG progenitors of type IIb, namely SN 1993J (Maund and Smartt (2009)), SN 2008ax (Folatelli et al. (2015)) and SN 2011dh (Van Dyk et al. (2013)). And one candidate SN 2013df (Van Dyk et al. (2014)). The YSG progenitor is difficult to explain by single stellar evolution models, unless mass-loss rates due to winds are assumed to be several times higher than the standard values (Georgy (2012)). On the contrary, a YSG star can be naturally produced in an interacting binary system (see e.g. Benvenuto et al. (2013)). Moreover, the possible detection of the companion of SN 1993J have been suggested by Maund et al. (2004) and more recent data appear to confirm this suggestion (Fox et al. (2014)). For the case of SN 2011dh, a blue point source with compatible properties as the predicted companion star has been detected in deep UV HST post-explosion images (Folatelli et al. (2014)). However, the optical postexplosion data of SN 2011dh are not conclusive about the companion detection (Maund et al. (2015)). Further observations need to be carried out in the coming years to confirm the association with the companion star.

Until very recently no firm progenitor identification was reported for H-deficient SNe (Eldridge et al. (2013)). The exception is SN iPTF13bvn, whose progenitor candidate was identified by Cao et al. (2013) and recently confirmed by Folatelli et al. (2016) and Eldridge \& Maund(2016) using HST post-explosion observations. Therefore, iPTF13bvn represents the first and until now only $\mathrm{H}$-deficient progenitor detected. At the moment no detection has been done of He-deficient SN Ic progenitors.

\section{Final Remarks}

Several pieces of evidence suggest that a large fraction of massive stars belong to interacting binary systems. This suggests that most SESNe arise from binaries. There is only one firm companion detection and possibly a second case.

Regarding progenitor masses, there is consistency between pre-explosion imaging and hydrodynamical modeling for H-poor objects. On the contrary, the situation is problematic among H-rich events. According to the pre-explosion imaging method for SNe II, initial masses of most "ordinary" CCSN progenitors seem to be $\lesssim 20 M_{\odot}$. This rises the question about what is the outcome of more massive stars. The current situation seems to indicate that efforts must be made in improving our understanding of massive star evolution.

\section{References}

Anderson J. P., Bersten, M. C., et al., in preparation

Anderson, J. P., Gutiérrez, C. P., Dessart, L., et al. 2016, A\&A, 590, C2

Anderson J. P., et al., 2014, ApJ, 786, 67

Benvenuto, O. G., Bersten, M. C., \& Nomoto, K. 2013, ApJ, 762, 74

Bersten, M. C., Benvenuto, O. G., Folatelli, G., et al. 2014, AJ, 148, 68

Bersten, M. C., Benvenuto, O. G., Nomoto, K., et al. 2012, ApJ, 757, 31

Bersten, M. C., Benvenuto, O., \& Hamuy, M. 2011, ApJ, 729, 61

Cao, Y., Kasliwal, M. M., Arcavi, I., et al. 2013, ApJl, 775, L7

Drout, M. R., Soderberg, A. M., Gal-Yam, A., et al. 2011, ApJ, 741, 97

Eldridge, J. J. \& Maund, J. R. 2016, MNRAS, 461, L117

Eldridge, J. J., Fraser, M., Smartt, S. J., Maund, J. R., \& Crockett, R. M. 2013, MNRAS, 436, 774

Filippenko, A. V. 1997, ARA $\& A, 35,309$

Folatelli, G., Van Dyk, S. D., Kuncarayakti, H., et al. 2016, ApJl, 825, L22

Folatelli, G., Bersten, M. C., et al. 2015, ApJ 
Folatelli, G., Bersten, M. C., Benvenuto, O. G., et al. 2014, ApJl, 793, L22

Fox, O. D., Azalee Bostroem, K., Van Dyk, S. D., et al. 2014, ApJ, 790, 17

Georgy, C. 2012, A\& A, 538, L8

González-Gaitán, S., Tominaga, N., Molina, J., et al. 2015, MNRAS, 451, 2212

Hamuy, M. \& Pinto, P. A. 2002, ApJl, 566, L63

Langer, N. 2012, ARA\&A, 50, 107

Lyman, J. D., Bersier, D., \& James, P. A. 2014, MNRAS, 437, 3848

Maund, J. R., Arcavi, I., Ergon, M., et al. 2015, MNRAS, 454, 2580

Maund, J. R. \& Smartt, S. J. 2009, Science, 324, 486

Maund, J. R., Smartt, S. J., Kudritzki, R. P., Podsiadlowski, P., \& Gilmore, G. F. 2004, Nature, 427,129

Morozova, V., Piro, A. L., \& Valenti, S. 2016, arXiv:1610.08054

Sana, H., de Mink, S. E., de Koter, A., et al. 2012, Science, 337, 444

Smartt, S. J. 2009, ARA\&A, 47, 63

Smartt, S. J. 2015, PASA, 32, e016

Smith, N., Li, W., Silverman, J. M., Ganeshalingam, M., \& Filippenko, A. V. 2011, MNRAS, 415,773

Utrobin, V. P. 2007, A\&A, 461, 233

Van Dyk, S. D., Zheng, W., Fox, O. D., et al. 2014, AJ, 147, 37

Van Dyk, S. D., Zheng, W., Clubb, K. I., et al. 2013, ApJ, 772, L32 